\title{
Posterior reversible encephalopathy syndrome in a patient with mixed connective tissue disease: a case report
}

\author{
Reza Rahmanzadeh ${ }^{1}$, Ramin Rahmanzade ${ }^{2}$ and Mozhdeh Zabihiyeganeh ${ }^{3^{*}}$
}

\begin{abstract}
Background: Posterior reversible encephalopathy is a syndrome highly associated with hypertension and cytotoxic therapy. The syndrome typically presents with headache, visual abnormality, seizures and characteristic vasogenic edema on magnetic resonance imaging. The entity warrants a prompt diagnosis to avoid deteriorating consequences.

Case presentation: In this report, we describe a 15-year-old Iranian boy who was diagnosed with mixed connective tissue disease, and cyclophosphamide pulse therapy was administered. Three days after the second pulse of cyclophosphamide, when he was receiving prednisolone and hydroxycholoroquine, our patient developed generalized tonic-clonic seizures. Magnetic resonance imaging findings showed high signal intensities in the posterior areas of his brain. After 8 days, the brain magnetic resonance imaging abnormalities were resolved following the control of his blood pressure and antiepileptic treatment. These observations have been indicative of posterior reversible encephalopathy syndrome. Nevertheless, our patient developed uncontrollable respiratory distress and eventually died.

Conclusions: To the best of our knowledge, this case is the first report of posterior reversible encephalopathy syndrome in a patient with mixed connective tissue disease. As the patient developed posterior reversible encephalopathy syndrome 3 days after cyclophosphamide pulse therapy to reduce the disease activity, it is hard to accurately determine whether posterior reversible encephalopathy syndrome in this case is a complication of cyclophosphamide or a condition that resulted from the mixed connective tissue disease flare-up.
\end{abstract}

Keywords: Posterior reversible encephalopathy syndrome, Mixed connective tissue disease, Cyclophosphamide

\section{Background}

Posterior reversible encephalopathy syndrome (PRES) is a life-threatening condition which can be characterized by symmetric involvement of posterior white matter on magnetic resonance imaging (MRI) and neurological impairments such as seizures, altered mental status, headache, and visual disturbances [1,2].

PRES has been reported in different conditions such as hypertensive encephalopathy, eclampsia, thrombotic thrombocytopenia purpura, and rheumatologic disorders [3-5].

The mainstay of management of PRES is timely diagnosis and discontinuation of causative agents that may

\footnotetext{
* Correspondence: Mozhdehzabihi@gmail.com

${ }^{3}$ Bone and Joint Reconstruction Research Center, Shafa Orthopedic Hospital,

Iran University of Medical Sciences, Tehran, Iran

Full list of author information is available at the end of the article
}

prevent subsequent abnormalities of the central nervous system.

The extensive use of immunosuppressive therapy and the autoimmune nature of rheumatologic diseases may make patients more vulnerable for developing PRES in the course of disease. Nevertheless, to the best of our knowledge, PRES has not been reported as a complication of treatment or a manifestation of disease in patients with mixed connective tissue disease (MCTD).

In this report, we describe a 15 -year-old Iranian boy with MCTD who presented with PRES 3 days after cyclophosphamide pulse therapy when he was receiving a high dose of steroids. Our patient was treated with antihypertension and antiepileptic medications and a repeat MRI scan showed no abnormality 8 days later. 


\section{Case presentation}

Our patient was a 15-year-old Iranian boy with a 2-year history of skin ulcer compatible to pyoderma gangrenosum. From the onset of his skin problems, he had been receiving a low dose of steroids, which was increased to 1 $\mathrm{mg} / \mathrm{kg} 2$ months prior to admission. He was referred to our hospital following development of muscle weakness and severe dyspnea. History-taking revealed a 1-year history of discoloration of his fingers in cold temperatures. A physical examination showed scleroderma-like signs of acrosclerosis and a small mouth orifice with difficulty in opening. Blood tests showed a remarkable elevation of muscle enzymes (creatine phosphokinase $[\mathrm{CPK}]>3000$, aldolase 39.4 and lactate dehydrogenase [LDH] 1510) and electromyogram-nerve conduction (EMG-NCV) tests indicated chronic moderate to severe myopathic process. We performed a muscle biopsy of his left deltoid muscle that revealed multiple necrotic fibers and extensive inflammatory endomysial foci. The laboratory findings showed antinuclear antibodies (ANA) 1:2500 positive, anti-doublestranded (ds) DNA 198 positive, anti-SM $>200$ positive, anti-SCL-70 $>200$ positive, anti-centromere $>2$ positive, anti-U1 RNP 178.4 positive, white blood cells (WBC) (from 3500 to 6900 during the hospitalization), hemoglobin $(\mathrm{Hb})$ 12.3, platelets (PLT) 128,000, and erythrocyte sedimentation rate (ESR) 56 (normal range $<30$ ). His anticardiolipin, anti-beta 2 glycoprotein I and lupus anticoagulant antibodies were negative and also his complement 3 (C3) and complement 4 (C4) levels were in normal range. Our patient fulfilled the Alarcon-Segovia diagnostic criteria [6] with positive serology and three of the five clinical criteria especially Raynaud's phenomenon, acrosclerosis, and myositis. Our patient also met the Kasukawa diagnostic criteria [6] with one common symptom of Raynaud's, positive serology, and mixed findings of leukopenia/thrombocytopenia, acrosclerosis, and muscle weakness. A chest X-ray showed diffuse pulmonary infiltration and a computed tomography (CT) scan reported a bronchiolitis obliterans organizing pneumonia (BOOP) reaction. Further tests also showed heart failure (ejection fraction $[\mathrm{EF}]=30 \%$ ) and pulmonary arterial hypertension (pulmonary artery pressure $[\mathrm{PAP}]=75 \mathrm{mmHg}$ ). Pulmonary embolus was ruled out by $\mathrm{CT}$ angiography. According to the criteria, our patient was diagnosed with mixed connective tissue disorder (MCTD), and $1 \mathrm{~g}$ intravenous methylprednisolone was administered. Then our patient received 500 mg cyclophosphamide pulse therapy, and was discharged with prednisolone $70 \mathrm{mg}$ daily and hydroxycholoroquine $200 \mathrm{mg}$ daily. Our patient received the second pulse of $500 \mathrm{mg}$ cyclophosphamide 2 weeks later. Three days after the second cyclophosphamide pulse when he was receiving prednisolone $70 \mathrm{mg} /$ day, he developed several generalized tonic-colonic seizures.
After admission to the intensive care unit, he developed another seizure that lasts 3 minutes. At this time, his blood pressure was 170/130. Therefore, phenytoin and antihypertension drugs were prescribed. An MRI scan of our patient revealed high signal intensities on T2weighted images and fluid-attenuated inversion recovery (FLAIR) sequences in the subcortical white matter of the occipital, posterior parietal, and posterior temporal lobes, and the cerebellum (Fig. 1). After 8 days, the brain MRI abnormalities had completely been resolved. These observations have been indicative of PRES.

From the first day of his second admission, our patient presented fever, cough and dyspnea and the laboratory tests showed creatinine $2.35 \mathrm{mg} / \mathrm{dL}$, urea $182 \mathrm{mg} / \mathrm{dL}$, WBC 3500, and platelets 75,000. A chest X-ray revealed extensive pleural effusion. In a peripheral blood smear, there was no evidence of thrombotic thrombocytopenia purpura. The serial blood test showed a progressive increase in his creatinine level and decrease in his platelet count. Therefore, we started rituximab $500 \mathrm{mg}$ for 2 weeks to control the disease flare-up. However, rituximab was not efficient and we started intravenous immunoglobulin (IV Ig) for 5 days. IV Ig did not improve our patient and we had to initiate plasma exchange. However, his creatinine level increased and respiratory symptoms became worse. Dialysis prevented the worsening of his condition and relieved our patient's symptoms. The results from bronchoalveolar lavage (BAL) showed infection to cytomegalovirus (CMV), Gram-negative bacillus, and candida. Although our patient received broad-spectrum antibiotics and antifungal agents, his respiratory manifestations were not improved and he was intubated. After 5 days of intubation, our patient developed heart arrest and, following 45 minutes of cardiopulmonary resuscitation (CRP), our patient died.

\section{Discussion}

Posterior reversible encephalopathy syndrome was first introduced by Hinchey and his colleagues in a study of 15 patients [7].

PRES can be diagnosed in a patient with reversible neurological manifestations including headache, nausea/ vomiting, visual abnormalities, consciousness impairment, seizure activity, and focal neurologic signs, in conjunction with bilateral involvement of posterior brain areas on magnetic resonance imaging [2].

The pathophysiology of PRES remains elusive. However, it has been suggested that a compromised cerebrovascular autoregulation due to acute hypertension may play a pivotal role. Accordingly, impaired cerebrovascular regulation may lead to arteriole leakage and cerebral vasogenic edema $[1,2]$. Although the co-occurrence of hypertension and PRES is remarkable, other possible 

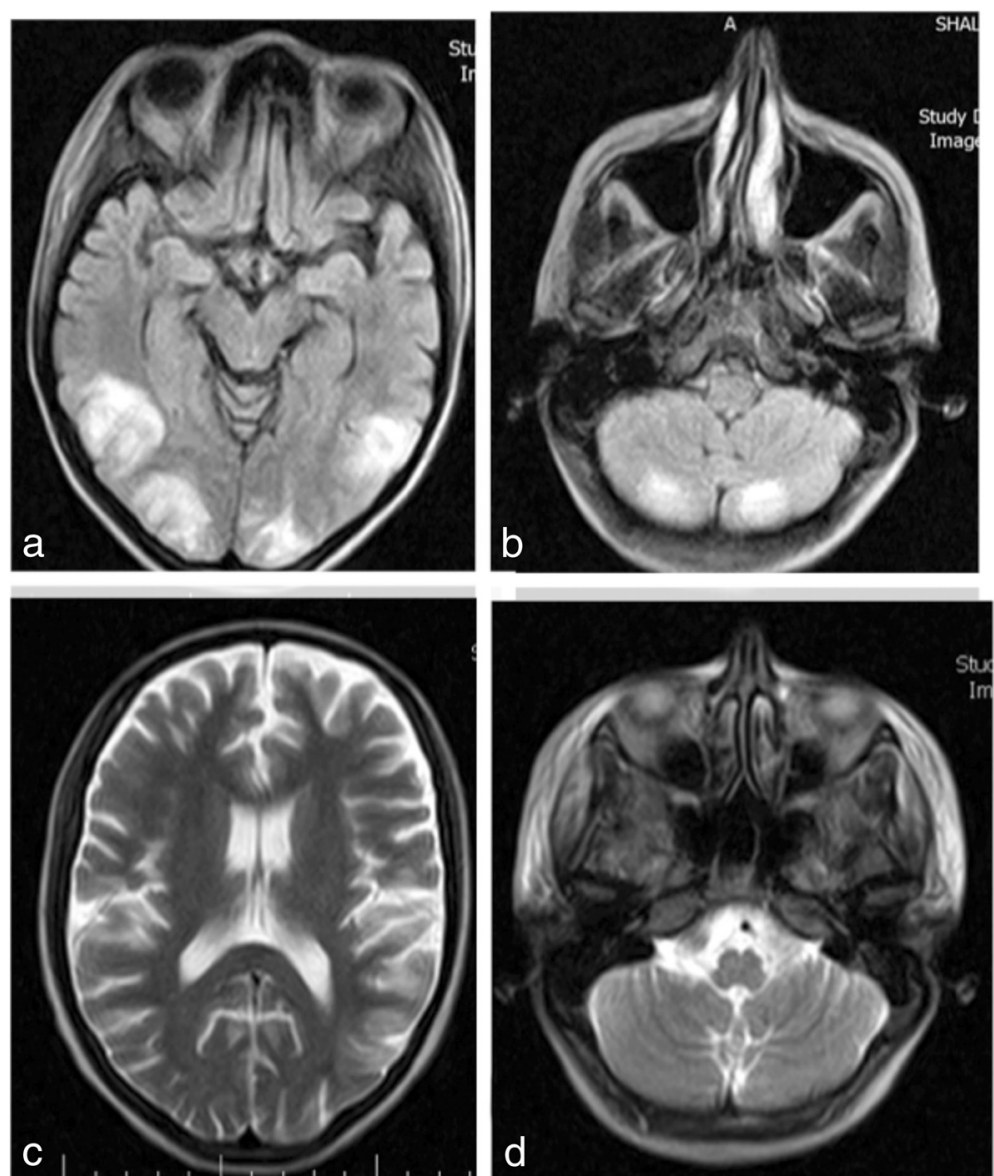

Fig. 1 Brain magnetic resonance imaging T2-weighted/fluid-attenuated inversion recovery scan showing high signal intensities in a the subcortical white matter of occipital, posterior parietal, and posterior temporal lobes and $\mathbf{b}$ the cerebellum. $\mathbf{c}$, $\mathbf{d}$ Follow-up brain magnetic resonance imaging T2-weighted/fluid-attenuated inversion recovery scan 8 days after the first imaging showed complete resolution

mechanisms have also been reported to be important in PRES such as disrupted cerebral autoregulatory mechanisms due to autonomic dysfunction [8], breakdown of blood-brain barrier following cytotoxic agents-induced endothelial toxicity, autoimmunity, and sepsis [9].

In addition, immunosuppressive agents such as methylprednisolone, dexamethasone, cyclosporine and cyclophosphamide have been reported to be related to PRES [10].

Our patient presented PRES 3 days after cyclophosphamide pulse therapy when he was receiving a high dose of prednisolone in the setting of a MCTD flare-up. On the other hand, clinical signs showed that our patient developed sepsis concomitant with PRES. Therefore, we could not accurately determine the main causative source of PRES among the cyclophosphamide pulse therapy, prednisolone, sepsis, and the flare-up of the underlying disease. Our patient had a 2-year history of taking steroids for his dermatological disorder that may decrease the importance of maintenance prednisolone as the cause of PRES. Most of the reported cases of PRES are suggested to be due to cytotoxic or steroid therapy. In the present case, the diagnosis of PRES a few days following the second pulse of cyclophosphamide may underscore its offensive effects and may suggest the cyclophosphamide pulse as a potential cause. Nevertheless, several reports show presentation of PRES unattributable to therapy in patients with rheumatologic diseases, which suggested it to be a condition resulting from underlying disease and even, in some cases, PRES was the first manifestation of the disease [11]. As in our case, PRES was concomitant with sepsis, therefore sepsis may play a role in this condition.

From the standpoint of pathogenesis, MCTD may be strongly suggestive as a cause of PRES. Various studies highlight that endothelial cell damage resulted from different autoantibodies in MCTD [12]. Along these lines, vasculopathy has been suggested to be a specific feature of MCTD that may lead to pulmonary arterial hypertension, which is responsible for most of the deaths in the late stages of MCTD [13]. In addition, the dysfunction of the autonomic system has been shown in MCTD [14]. Therefore, PRES may be a manifestation of MCTD 
in our case because our patient presented PRES during the disease flare-up.

To the best of our knowledge, a case of PRES in a patient with mixed connective tissue disease has not yet been reported. The neurological manifestations of MCTD have been believed to be less frequent than findings of other systems. Although the main neurological manifestations of MCTD are trigeminal neuropathy, headaches, and aseptic meningitis, this report suggests PRES as a neurological condition which may occur during the course of MCTD [15]. Although there is no difference between our patient and previously reported cases in terms of PRES characteristics, further reports are needed for better understanding of PRES in MCTD.

\section{Conclusions}

To the best of our knowledge, this case is the first report of posterior reversible encephalopathy syndrome in a patient with mixed connective tissue disease. As the patient developed PRES shortly after the cyclophosphamide pulse, during an MCTD flare-up, and concomitant with sepsis, it is hard to determine the accurate cause of PRES in this case. Taken together, MCTD may be strongly suggestive as the cause of PRES for the extensive endothelial dysfunction involved in its pathogenesis.

\section{Consent}

Written informed consent was obtained from the patient's family for publication of this case and any accompanying images. A copy of the written consent is available for review by the Editor-in-Chief of this journal.

\section{Abbreviations}

$C T$, computed tomography; MCTD, mixed connective tissue disease; MRI, magnetic resonance imaging; PRES, posterior reversible encephalopathy syndrome.

\section{Acknowledgements}

The authors thank the family of the patient for their assistance in preparing the manuscript.

\section{Authors' contributions}

$R R, R R$, and MZY prepared the manuscript and collected all data and suitable references. All authors read and approved the final manuscript.

\section{Competing interests}

The authors declare that they have no competing interests.

\section{Author details}

'Division of Neuroscience, Cellular and Molecular Research Center, Iran University of Medical Sciences, Tehran, Iran. ${ }^{2}$ Division of Neuroscience, Neurology Research Center, Shahid Beheshti University of Medical Sciences, Tehran, Iran. ${ }^{3}$ Bone and Joint Reconstruction Research Center, Shafa Orthopedic Hospital, Iran University of Medical Sciences, Tehran, Iran.

Received: 6 January 2016 Accepted: 18 May 2016

Published online: 02 June 2016

\section{References}

1. Bartynski WS. Posterior reversible encephalopathy syndrome, part 2: controversies surrounding pathophysiology of vasogenic edema. AJNR Am J Neuroradiol. 2008:29(6):1043-9.

2. Bartynski WS. Posterior reversible encephalopathy syndrome, part 1: fundamental imaging and clinical features. AJNR Am J Neuroradiol. 2008;29(6):1036-42.

3. Schaefer PW, Buonanno FS, Gonzalez RG, Schwamm LH. Diffusion-weighted imaging discriminates between cytotoxic and vasogenic edema in a patient with eclampsia. Stroke. 1997;28(5):1082-5.

4. Provenzale JM, Petrella JR, Cruz Jr LC, Wong JC, Engelter S, Barboriak DP. Quantitative assessment of diffusion abnormalities in posterior reversible encephalopathy syndrome. AJNR Am J Neuroradiol. 2001;22(8):1455-61.

5. Covarrubias DJ, Luetmer PH, Campeau NG. Posterior reversible encephalopathy syndrome: prognostic utility of quantitative diffusionweighted MR images. AJNR Am J Neuroradiol. 2002;23(6):1038-48.

6. Amigues JM, Cantagrel A, Abbal M, Mazieres B. Comparative study of 4 diagnosis criteria sets for mixed connective tissue disease in patients with anti-RNP antibodies. Autoimmunity Group of the Hospitals of Toulouse. J Rheumatol. 1996;23(12):2055-62.

7. Hinchey J, Chaves C, Appignani B, Breen J, Pao L, Wang A, et al. A reversible posterior leukoencephalopathy syndrome. N Engl J Med. 1996;334(8):494-500.

8. Joa KL, Shin Yl, Suh H, Kim SY, Chang JH, Ko HY. Autonomic dysreflexiainduced reversible posterior leukoencephalopathy syndrome in patients with spinal cord injury: two case reports. I Spinal Cord Med. 2013;36(3):250-3.

9. Bartynski WS, Boardman JF, Zeigler ZR, Shadduck RK, Lister J. Posterior reversible encephalopathy syndrome in infection, sepsis, and shock. AJNR Am J Neuroradiol. 2006;27(10):2179-90.

10. McKinney AM, Short J, Truwit CL, McKinney ZJ, Kozak OS, SantaCruz KS, et al. Posterior reversible encephalopathy syndrome: incidence of atypical regions of involvement and imaging findings. AJR Am J Roentgenol. 2007;189(4):904-12.

11. Barber CE, Leclerc R, Gladman DD, Urowitz MB, Fortin PR. Posterior reversible encephalopathy syndrome: an emerging disease manifestation in systemic lupus erythematosus. Semin Arthritis Rheum. 2011:41(3):353-63.

12. Soltesz P, Bereczki D, Szodoray P, Magyar MT, Der H, Csipo I, et al. Endothelial cell markers reflecting endothelial cell dysfunction in patients with mixed connective tissue disease. Arthritis Res Ther. 2010;12(3):R78.

13. Bull TM, Fagan KA, Badesch DB. Pulmonary vascular manifestations of mixed connective tissue disease. Rheum Dis Clin North Am. 2005;31(3):451-64. vi.

14. Stacher G, Merio R, Budka C, Schneider C, Smolen J, Tappeiner G. Cardiovascular autonomic function, autoantibodies, and esophageal motor activity in patients with systemic sclerosis and mixed connective tissue disease. J Rheumatol. 2000;27(3):692-7.

15. Ortega-Hernandez OD, Shoenfeld Y. Mixed connective tissue disease: an overview of clinical manifestations, diagnosis and treatment. Best Pract Res Clin Rheumatol. 2012;26(1):61-72.

Submit your next manuscript to BioMed Central and we will help you at every step:

- We accept pre-submission inquiries

- Our selector tool helps you to find the most relevant journal

- We provide round the clock customer support

- Convenient online submission

- Thorough peer review

- Inclusion in PubMed and all major indexing services

- Maximum visibility for your research 